\title{
Experiments in Iterative Feedback Tuning for Level Control of Three-Tank System
}

\author{
Radu-Emil Precup ${ }^{\# 1}$, Ioan Mosincat ${ }^{* 2}$, Mircea-Bogdan Radac ${ }^{\# 3}$, Stefan Preitl ${ }^{\# 4}$, Stefan Kilyeni ${ }^{\# 5}$, Emil M. Petriu ${ }^{\# \# 6}$, \\ Claudia-Adina Dragos ${ }^{\# 7}$ \\ \# Department of Automation and Applied Informatics, "Politehnica” University of Timisoara \\ Bd. V. Parvan 2, 300223 Timisoara, Romania \\ ${ }^{1}$ radu.precup@aut.upt.ro \\ 3 mircea.radac@aut.upt.ro \\ ${ }^{4}$ stefan.preit1@aut.upt.ro \\ ${ }^{5}$ stefan.kilyeni@et.upt.ro \\ claudia.dragos@aut.upt.ro \\ * Department of Energy Technology, Aalborg University \\ Pontoppidanstrcede 101, DK-9220 Aalborg East, Denmark \\ 2 ionicam@gmail.com \\ \# School of Information Technology and Engineering, University of Ottawa \\ 800 King Edward, Ottawa, Ontario, K1N 6N5, Canada \\ ${ }^{6}$ petriu@site.uottawa.ca
}

\begin{abstract}
This paper investigates the applicability of Iterative Feedback Tuning (IFT) to the level control of a three-tank system laboratory equipment. First two PID controllers are designed in terms of the frequency domain approach. Next IFT is employed to improve the control system performance indices. Digital simulation results and real-time experimental results validate the new control solution.
\end{abstract}

\section{INTRODUCTION}

The design of optimal control system (CS) structures is very important when very good CS performance indices are required. Therefore the minimization of the objective functions (o.f.s) [1]-[8] as part of appropriately defined optimization problems can ensure the fulfillment of the required CS performance indices if the o.f.s are expressed as integral quadratic performance indices. The Iterative Feedback Tuning (IFT) [9], [10], which performs a gradient-based minimization of the o.f.s, provides an efficient way to deal with some of the specific problems occurring in the case of the nonlinear or ill-defined processes.

IFT offers a model-free direct data-based offline-adaptive controller design approach based on some experiments per iteration done with the closed-loop CS. The extension of IFT according to [11] provides additional data to improve the convergence properties of IFT aiming the disturbance rejection. Extensions of IFT to Multi Input-Multi Output (MIMO) systems are discussed in [12]-[15]. Linear applications applied to digitally simulated benchmarks are presented in [12], [13]. The need for faster gradient approximations together with the local convergence in ITF for MIMO processes are analyzed in [14]. Recently reported IFT applications to industrial control problems deal with chemical servo drives [15], [16], and chemical processes [17].

The three-tank system (3TS) is an important benchmark that can illustrate several problems of control design, fault detection and diagnosis. The current approaches to the level control of 3TS concern the popular PID control [18], robust and fuzzy predictive control [19], [20], nonlinear backstepping [21], or sliding mode control [22].

In this context, this paper has two main contributions. First, a systematic application of the IFT to the level control of the 3TS is presented and a new control solution is thus suggested. Second, digital simulation results and real-time experimental results for the AMIRA DTS 200 3TS laboratory equipment [23] are included to validate the new control solutions.

The paper treats the following topics. The mathematical model of the controlled process and the CS structure are presented in Section II. Next and overview on IFT is sketched in Section III. Section IV presents the application of the IFT to the level control of the 3TS and suggests an original low-cost control solution validated by digital simulation and real-time experimental results. The conclusions are drawn in Section V.

\section{Controlled Process AND Control System STRUCTURE}

The controlled process (Fig. 1, Fig. 2) consists of three identical cylindrical tanks T1, T2 and T3, connected serially with each other by cylindrical pipes. The liquid, which is collected in a reservoir placed beneath the tanks, is pumped into the tanks T1 and T2 by two variable speed pumps driven by DC motors, playing the role of actuators fed by the control signals $u_{1}$ and $u_{2}$, respectively, which stand for the voltages applied to the DC motors. All tanks are equipped with static pressure sensors which give the voltage outputs $u_{h i}$, proportional to the three liquid levels $h_{i}, i=\overline{1,3}$. The leak valves $\alpha_{j}, j \in\{e 1, e 2, e 3,31,32, g 2\}$, drain manually each tank. 


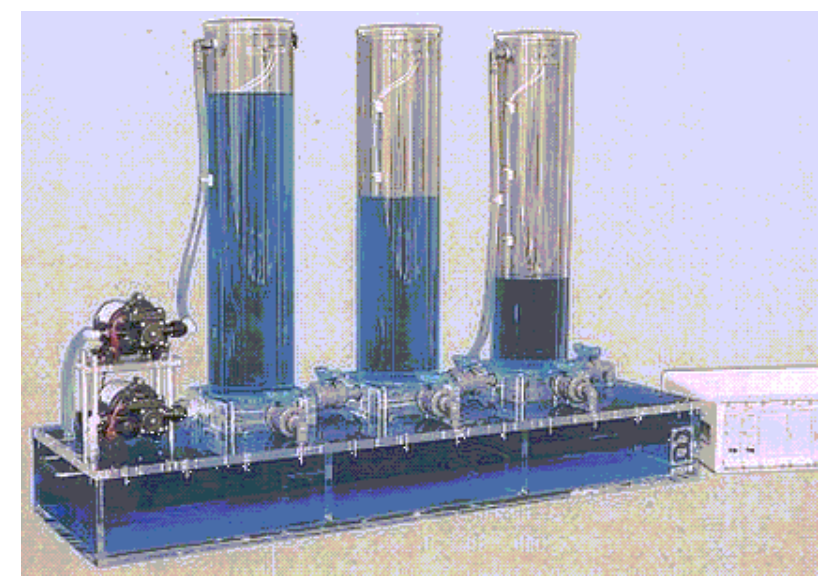

Fig. 1. Amira DTS 200 3TS laboratory equipment experimental setup

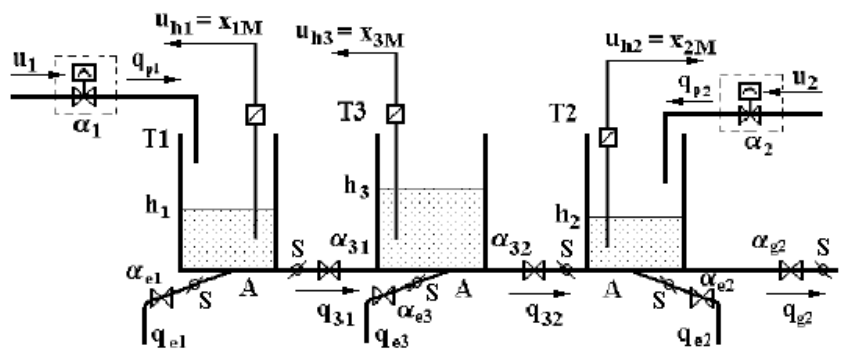

Fig. 2. Structure of controlled process

The first principle state-space mathematical model of the process [18], [23], [24] is a nonlinear third-order system with all parameters having physical meaning. The following first principle model can be derived from the balance equations and Torricelli's law:

$$
\begin{aligned}
& \dot{h}_{1}=-(1 / A) \mu_{s 1} S \operatorname{sgn}\left(h_{1}-h_{3}\right) \sqrt{2 g\left|h_{1}-h_{3}\right|}- \\
& -(1 / A) \mu_{e 1} S \sqrt{2 g h_{1}}+(1 / A) c_{1} u_{1}, \\
& \dot{h}_{2}=(1 / A) \mu_{s 1} S \operatorname{sgn}\left(h_{3}-h_{2}\right) \sqrt{2 g\left|h_{3}-h_{2}\right|}- \\
& -(1 / A) \mu_{e 2} S \sqrt{2 g h_{2}}-(1 / A) \mu_{g 2} S \sqrt{2 g h_{2}}+(1 / A) c_{1} u_{2}, \\
& \dot{h}_{3}=(1 / A) \mu_{s 1} S \operatorname{sgn}\left(h_{1}-h_{3}\right) \sqrt{2 g\left|h_{1}-h_{3}\right|}- \\
& -(1 / A) \mu_{s 2} S \operatorname{sgn}\left(h_{3}-h_{2}\right) \sqrt{2 g\left|h_{3}-h_{2}\right|}- \\
& -(1 / A) \mu_{e 3} S \sqrt{2 g h_{3}},
\end{aligned}
$$

where $A$ is the cross section of the tanks, $S$ is the cross section of the pipes, $c_{1}=q_{p 1} / u_{1}$ and $c_{2}=q_{p 2} / u_{2}$ are the gains of the two pumps, $0 \leq \mu_{s 1} \leq 1$ is the outflow coefficient between $\mathrm{T} 1$ and $\mathrm{T} 3,0 \leq \mu_{s 2} \leq 1$ is the outflow coefficient between T2 and $\mathrm{T} 3, \mu_{e 1}, \mu_{e 2}, \mu_{e 3}, \mu_{g 2}$ are the outflow coefficients within 0 (for completely open valve) and 1 (for completely closed valve) according to Fig. 2, and $g$ is the gravitational acceleration.

The typical control objectives concerning the level control in the 3TS are to keep the desired liquid levels in the tanks T1 and T2, viz. $h_{1}$ and $h_{2}$, as specified by the two reference inputs $r_{1}$ and $r_{2}$, respectively, and do the rejection of six possible disturbances (gathered in the disturbance input vector d) due to the six manual valves. The level in the tank $\mathrm{T} 3, h_{3}$, is a response which is uncontrollable.

A low-cost control solution that that can fulfill the control objectives makes use of the CS structure presented in Fig. 3, where $u_{h 1}$ and $u_{h 2}$ are the measured levels corresponding to $h_{1}$ and $h_{2}$, respectively, $e_{1}=r_{1}-h_{1}$ and $e_{2}=r_{2}-h_{2}$ are the control errors, $\mathbf{d}=\left[\begin{array}{llll}\mu_{e 1} & \mu_{e 2} & \mu_{e 3} & \mu_{g 2}\end{array}\right]^{T}$.

Fig. 3 points out a MIMO CS structure with two control loops. The low-cost control solution can be designed if the following two assumptions hold:

- There is no need for decoupling controllers because it is considered that the two channels (corresponding to $h_{1}$ and $h_{2}$ ) represent additional disturbances (with the same roles as $\mathbf{d}$ ).

- The low-cost controllers with the transfer functions $C_{1}(s)$ and $C_{2}(s)$ are designed separately making use of the linearized mathematical model of the controlled process which is valid for $S / A<<1$.

Accepting the two assumptions the following linearized state-space mathematical model of the controlled process can be derived around the equilibrium point characterized by the superscript 0 inserted to the variables:

$$
\begin{aligned}
& \Delta \dot{h}_{1}=-(S / A) \operatorname{sgn}\left(h_{1}-h_{3}\right)\left[\sqrt{2 g\left|h_{10}-h_{30}\right|} \Delta \mu_{s 1}+\right. \\
& \left.+\mu_{s 10} \sqrt{g /\left(2\left|h_{10}-h_{30}\right|\right)}\left(\Delta h_{1}-\Delta h_{3}\right)\right]- \\
& -(S / A) \sqrt{2 g h_{10}} \Delta \mu_{e 1}-(S / A) \mu_{e 10} \sqrt{g /\left(2 h_{10}\right)} \Delta h_{1}+ \\
& +\left(c_{1} / A\right) \Delta u_{1}, \\
& \Delta \dot{h}_{2}=(S / A) \operatorname{sgn}\left(h_{3}-h_{2}\right)\left[\sqrt{2 g\left|h_{30}-h_{20}\right|} \Delta \mu_{s 1}-\right. \\
& \left.-\mu_{s 10} \sqrt{g /\left(2\left|h_{30}-h_{20}\right|\right)}\left(\Delta h_{2}-\Delta h_{3}\right)\right]- \\
& -(S / A) \sqrt{g /\left(2 h_{20}\right)}\left(\mu_{e 20}+\mu_{g 20}\right) \Delta h_{2}- \\
& -(S / A) \sqrt{2 g h_{20}}\left(\Delta \mu_{e 2}+\Delta \mu_{g 2}\right)+\left(c_{1} / A\right) \Delta u_{2}, \\
& \dot{h}_{3}=(S / A) \operatorname{sgn}\left(h_{1}-h_{3}\right)\left[\sqrt{2 g\left|h_{10}-h_{30}\right|} \Delta \mu_{s 1}+\right. \\
& \left.+\mu_{s 10} \sqrt{g /\left(2\left|h_{01}-h_{30}\right|\right)}\left(\Delta h_{1}-\Delta h_{3}\right)\right]- \\
& -(S / A) \operatorname{sgn}\left(h_{3}-h_{2}\right)\left[\sqrt{2 g\left|h_{30}-h_{20}\right| \Delta} \mu_{s 2}-\right. \\
& -\mu_{s 20} \sqrt{g /\left(2\left|h_{30}-h_{20}\right|\right)}\left(\Delta h_{2}-\Delta h_{3}\right)- \\
& -(S / A)\left(\sqrt{2 g h_{30}} \Delta \mu_{e 3}+\mu_{e 30} \sqrt{g /\left(2 h_{30}\right)} \Delta h_{3},\right.
\end{aligned}
$$

where $\Delta w$ is generally the difference of the variable $w$ with respect to the coordinate of the equilibrium point.

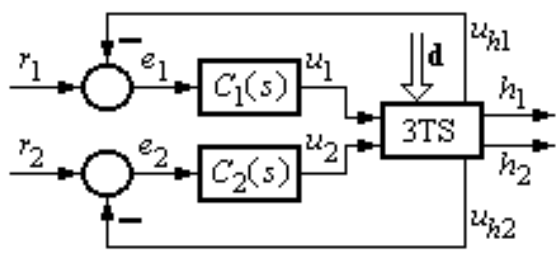

Fig. 3. Structure of control system 
The state-space mathematical model (2) permits the calculation of the transfer function matrix of the controlled process including the actuators and sensors. Accounting for the two assumptions mentioned above only the main diagonal elements of that matrix are important, and they are the transfer functions of the controlled processes in the two control loops containing the controllers $C_{1}(s)$ and $C_{2}(s)$.

\section{OVERVIEW ON ITERATIVE FEEDBACK TUNING}

The presentation is focused on only one of the two controllers in Fig. 3. Therefore the structure of the CS with IFT is presented in Fig. 4 (in the linear case), where: $r$ - the reference input, $d-$ the disturbance input (noise), $e-$ the control error, $u$ - the control signal, $\boldsymbol{\rho}-$ the parameter vector containing the controller parameters, $C(\boldsymbol{\rho})$ - the controller transfer function, $F$ - the reference model transfer function (however the CS is not a model reference adaptive control because the structure does not operate permanently) $P$ - the controlled process transfer function, $y$ - the controlled output, $y_{d}$ - the desired output (of reference model), $\delta y=y-y_{d}-$ the tracking error, and IFT - Iterative Feedback Tuning algorithm.

The input vector i contains several important variables related to the CS behavior i.e. the variables and parameters specific to the optimization problem mentioned in Section I. The operational variable in the transfer functions has been omitted to ensure simplicity, but it will be included as follows in the well-accepted notation $s$ for continuous-time and $z$ for discrete-time to improve the clarity; that is the reason why the argument $\boldsymbol{\rho}$ is inserted to some of CS variables. The controller parameterization should ensure the differentiable with respect to $\boldsymbol{\rho}$ transfer function $C(\boldsymbol{\rho})$. The controller must stabilize first the CS so the initial tuning of the controller is important as in case of all iterative methods. The expression of the o.f. $J(\boldsymbol{\rho})$ is

$$
J(\boldsymbol{\rho})=(0.5 / N) \sum_{k=1}^{N}[\delta y(k, \boldsymbol{\rho})]^{2}
$$

where $N$ is the number of samples / length of an experiment. General expressions of the o.f. that can weight the control signal are treated in [25], [26].

A typical objective concerning $J(\rho)$ is to find the optimal parameter vector $\boldsymbol{\rho}^{*}$ to minimize the o.f. and make the tracking error $\delta y$ tend to zero. That objective can be expressed in terms of the optimization problem

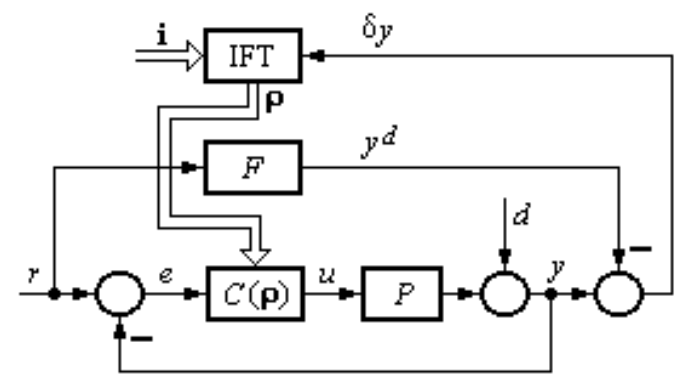

Fig. 4. Structure of control system with IFT

$$
\boldsymbol{\rho}^{*}=\underset{\boldsymbol{\rho} \in S C}{\arg \min } J(\boldsymbol{\rho}),
$$

where $S$ is the set of constraints imposed in relation with the process or CS. The stability analysis of the CS should be done with this regard.

IFT algorithms solve the optimization problem (4) by numerical optimization techniques making use of the control signal and controlled output during the CS operation. Newton's method is such a technique, which iteratively approaches a zero of a function without knowledge on its full expression. The update law by to calculate the next parameter vector $\boldsymbol{\rho}^{i+1}$ depending on the current one is

$$
\boldsymbol{\rho}^{i+1}=\boldsymbol{\rho}^{i}-\gamma_{i} \mathbf{R}_{i}^{-1} \frac{\partial J}{\partial \boldsymbol{\rho}}\left(\boldsymbol{\rho}^{i}\right)
$$

where the positive definite regular matrix $\mathbf{R}_{i}$ is typically a Gauss-Newton approximation of the Hessian of $J$, the identity matrix in the simplest case. The coefficient $\gamma_{i}$ is used to scale the default step size.

The IFT algorithm consists of the following steps, with the steps 1 to 5 repeated at each iteration:

- Step 0. Set the parameters in the o.f., the step size and the initial controller parameter vector $\boldsymbol{\rho}^{0}$.

- Step 1. Do the two experiments needed by one-degreeof-freedom controllers and record the input-output data pairs $\left(u_{1}, y_{1}\right)$ and $\left(u_{2}, y_{2}\right)$. The first experiment is called the normal one (the usual operation of the CS) and the second experiment is the gradient one. In the gradient experiment the reference input is the control error of the first one.

- Step 2. Calculate the estimates of the gradients of tracking error

$$
\frac{\partial \delta y}{\partial \boldsymbol{\rho}}\left(k, \boldsymbol{\rho}^{i}\right)=\frac{1}{C\left(q^{-1}, \boldsymbol{\rho}^{i}\right)} \cdot \frac{\partial C}{\partial \boldsymbol{\rho}}\left(q^{-1}, \boldsymbol{\rho}^{i}\right) \cdot y_{2}\left(k, \boldsymbol{\rho}^{i}\right),
$$

where the subscript 2 outlines the experiment index and $q^{-1}$ is the backward shift operator.

- Step 3. Generate $y_{d}$ and calculate $\delta y$.

- Step 4. Calculate the estimates of the gradient of $J$ making use of (6) substituted to (7):

$$
\frac{\partial J}{\partial \boldsymbol{\rho}}\left(\boldsymbol{\rho}^{i}\right)=(1 / N) \sum_{k=1}^{N}\left[\delta y\left(k, \boldsymbol{\rho}^{i}\right) \frac{\partial \delta y}{\partial \boldsymbol{\rho}}\left(k, \boldsymbol{\rho}^{i}\right)\right] \cdot
$$

- Step 5. Calculate the next set of parameters $\boldsymbol{\rho}^{i+1}$ by the update law (5).

The IFT algorithm is stopped when the o.f. has decreased enough showing a serious improvement of the CS performance indices. Therefore a quasi-optimal CS structure is given, and the CS will operate after the IFT with the new parameters $\boldsymbol{\rho}^{*}$. 
Only convergent IFT algorithms ensure the CS performance improvement. Therefore the convergence analysis should be accounted for.

\section{CASE STUDY}

The new control solution is based on two discrete-time PID controllers that can control the given process. The controller design starts with two continuous-time PID controllers with the transfer functions

$$
C_{h}(s)=k^{h}\left[1+1 /\left(s T_{i}^{h}\right)+s T_{d}^{h} /\left(s T_{d}^{h} / N^{h}+1\right)\right], i=\overline{1,2},
$$

where: $k^{h}$ - the controller gains, $T_{i}^{h}$ - the integral time constants and $T_{d}^{h}$ - the derivative time constants, $N^{h}$ - the maximum derivative gains, $h=\overline{1,2}$. A frequency domain design approach is applied in the step 1 of the IFT algorithm. Next the value of the sampling period $T_{s}$ is set according to the requirements of quasi-continuous digital control. Tustin's method employed for discretization results in the two discrete-time PID controllers

$$
\begin{aligned}
& C\left(q^{-1}, \boldsymbol{\rho}\right)=\frac{\rho_{0}^{h}+\rho_{1}^{h} q^{-1}+\rho_{2}^{h} q^{-2}}{1+\rho_{3}^{h} q^{-1}+\rho_{4}^{h} q^{-2}}, \\
& \boldsymbol{\rho}=\left[\begin{array}{llll}
\rho_{0}^{h} & \rho_{1}^{h} & \rho_{2}^{h} & \rho_{3}^{h}
\end{array}\right]^{T}, \\
& \rho_{0}^{h}=k^{h}\left[1+2 T_{d}^{h} N^{h} /\left(2 T_{d}^{h}+T_{s} N^{h}\right)\right] \text {, } \\
& \rho_{1}^{h}=k^{h}\left[-1+T_{d}^{h} /\left(2 T_{i}^{h}\right) T_{s}-4 T_{d}^{h} N^{h} /\left(2 T_{d}^{h}+T_{s} N^{h}\right)\right. \text {, } \\
& \rho_{2}^{h}=2 k^{h} T_{d}^{h} /\left(2 T_{d}^{h}+T_{s} N^{h}\right) \text {, } \\
& \rho_{3}^{h}=-4 T_{d}^{h} /\left(2 T_{d}^{h}+T_{s} N^{h}\right) \text {, } \\
& \rho_{4}^{h}=-1-\rho_{3}^{h}, h=\overline{1,2} \text {. }
\end{aligned}
$$

The estimates of the gradients of tracking error needed in the step 2 of the IFT algorithm result as follows making use of (6) and (9):

$$
\begin{aligned}
& \frac{\partial \delta y}{\partial \boldsymbol{\rho}}\left(k, \boldsymbol{\rho}^{i}\right)=\left[\begin{array}{c}
1 / A\left(q^{-1}, \boldsymbol{\rho}^{i}\right) \\
q^{-1} / A\left(q^{-1}, \boldsymbol{\rho}^{i}\right) \\
q^{-2} / A\left(q^{-1}, \boldsymbol{\rho}^{i}\right) \\
q^{-1} / B\left(q^{-1}, \boldsymbol{\rho}^{i}\right)
\end{array}\right] y_{2}\left(k, \boldsymbol{\rho}^{i}\right), \\
& A\left(q^{-1}, \boldsymbol{\rho}^{i}\right)=\left(\rho_{0}^{h}\right)^{i}+\left(\rho_{1}^{h}\right)^{i} q^{-1}+\left(\rho_{2}^{h}\right)^{i} q^{-2}, \\
& B\left(q^{-1}, \boldsymbol{\rho}^{i}\right)=\left(\rho_{0}^{h}\right)^{i}+\left(\rho_{3}^{h}\right)^{i} q^{-1}+\left(\rho_{4}^{h}\right)^{i} q^{-2}, h=\overline{1,2} .
\end{aligned}
$$

Making use of the parameter values of the controlled process given in [23], [24], the controlled process can be reduced to second-order systems for each of the two control loops. Therefore the reference model applied in the step 3 of the IFT algorithm can be set in terms of

$$
F(s)=1 /\left(s^{2}+s+1\right),
$$

which is next discretized for $T_{s}=1 \mathrm{~s}$. Having these elements, the application of the steps 3 to 5 of the IFT algorithm is straightforward.
Accepting the symmetry of the levels corresponding to the equilibrium point the two transfer functions of the controlled processes are identical and they result in identical PID controllers. The frequency domain design imposing a phase margin of $45^{\circ}$ followed by the application of (9) leads to the initial transfer function of the discrete-time PID controllers

$$
C\left(q^{-1}, \boldsymbol{\rho}^{0}\right)=\frac{86.6667-153 q^{-1}+66.6667 q^{-2}}{1-1.3333 q^{-1}+0.3333 q^{-2}} .
$$

The behavior of the CS before IFT with respect to the step type modifications $r_{1}=0.25 \mathrm{~m}$ and $r_{1}=0.15 \mathrm{~m}$ of the reference inputs is presented in Fig. 5 (a). A band-limited white noise with zero mean and variance $\sigma^{2}=0.002$ was added to the controlled outputs during the digital simulation of the CS behavior.

The matrix $\mathbf{R}_{i}$ was set equal to $\mathbf{I}_{4}$. The step size was scaled by $\gamma_{i}=10^{-4}$ so that the changes in the parameter should not affect stability of the CS. The PID controllers obtain the following expression after 4 iterations of the IFT algorithm:

$$
C\left(q^{-1}, \boldsymbol{\rho}^{4}\right)=\frac{66.2469-132.538 q^{-1}+66.6323 q^{-2}}{1-1.4093 q^{-1}+0.5907 q^{-2}} .
$$

and the behavior of the CS is presented in Fig. 5 (b).

Fig. 5 (b) shows the improvement of the CS performance indices settling time and overshoot. However the improvement is still not enough. Therefore the IFT algorithm is continued, and the PID controllers obtain the expressions (11) and (12) after 8 and 12 iterations, respectively, of the IFT algorithms:

$$
C\left(q^{-1}, \boldsymbol{\rho}^{8}\right)=\frac{66.247-132.5382 q^{-1}+66.6323 q^{-2}}{1+0.0295 q^{-1}-1.0295 q^{-2}},
$$
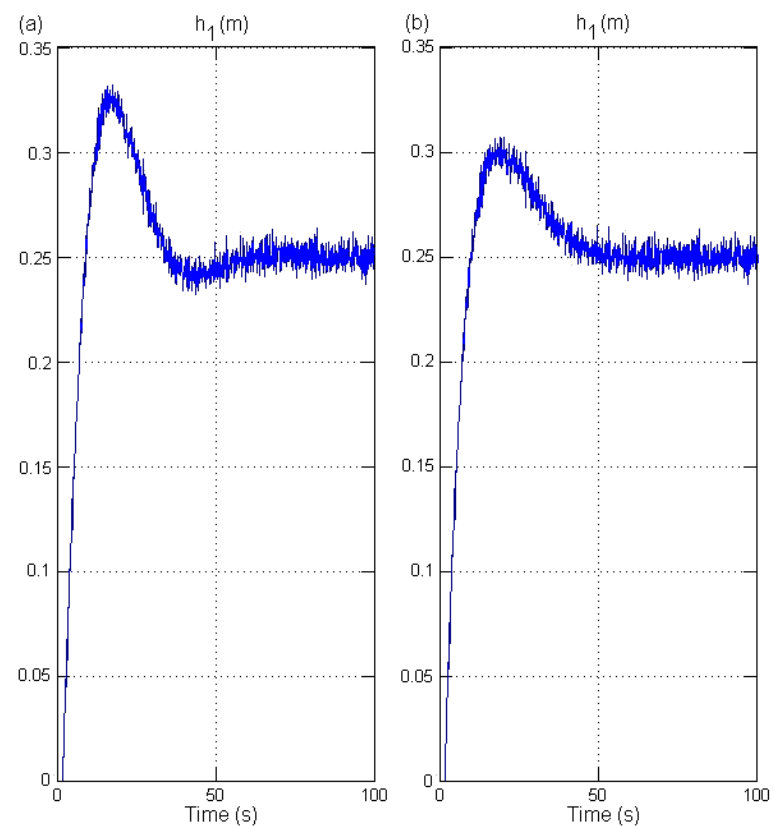

Fig. 5. Digital simulation results: $h_{1}$ versus time before IFT (a), after 4 iterations of IFT algorithm (b) 


$$
C\left(q^{-1}, \boldsymbol{\rho}^{12}\right)=\frac{66.2472-132.5387 q^{-1}+66.6326 q^{-2}}{1+0.0293 q^{-1}-1.0293 q^{-2}},
$$

with the behaviors of the CS illustrated in Fig. 6 (a) and (b), respectively. A serious improvement of the CS performance indices can be observed.

The evolution of the o.f. $J$ over the iterations is presented in Fig. 7. It is shown that the application of the IFT algorithm after 10 iterations is useless. Therefore the IFT algorithm is stopped according to the previous section.

After 12 iterations part of the real-time experimental results with the CS designed by IFT is presented in Figs. 8 to 10. The same reference inputs as in the digital simulations were applied. The unit step modification of the disturbance input $\mu_{e 1}$ after $50 \mathrm{~s}$ and the unit step modification of the disturbance input $\mu_{g_{2}}$ after $150 \mathrm{~s}$ were applied manually by the complete opening of the corresponding valves (Fig. 2). The real-time experimental results are different to the simulation ones, the oscillations are present because of the waves caused by the pumps during the process of tank filling. As mentioned before, the level $h_{3}$ is not important being just a response.
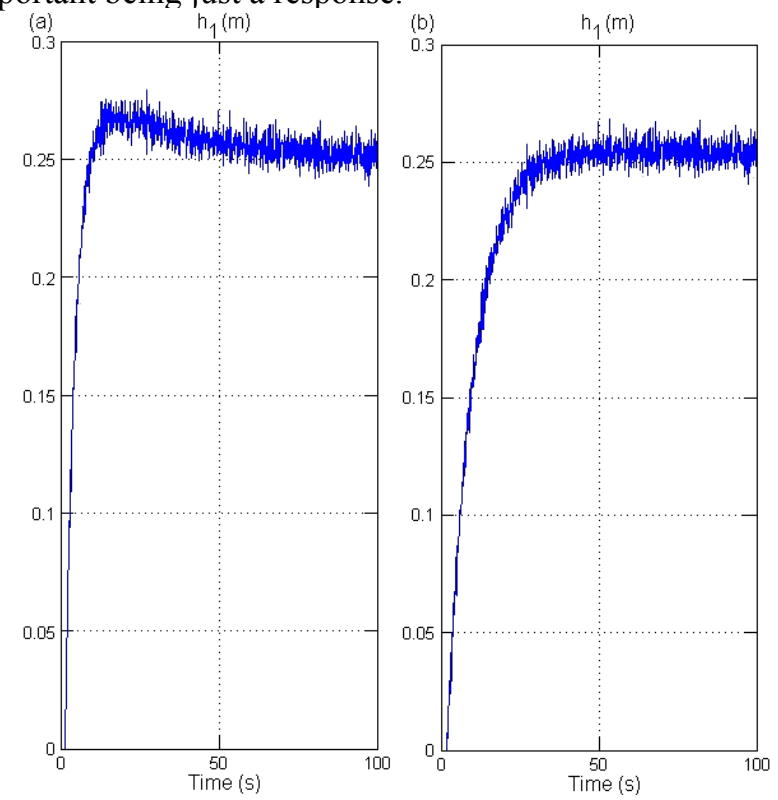

Fig. 6. Digital simulation results: $h_{1}$ versus time after 8 iterations (a) and 12 iterations (b) of the IFT algorithm

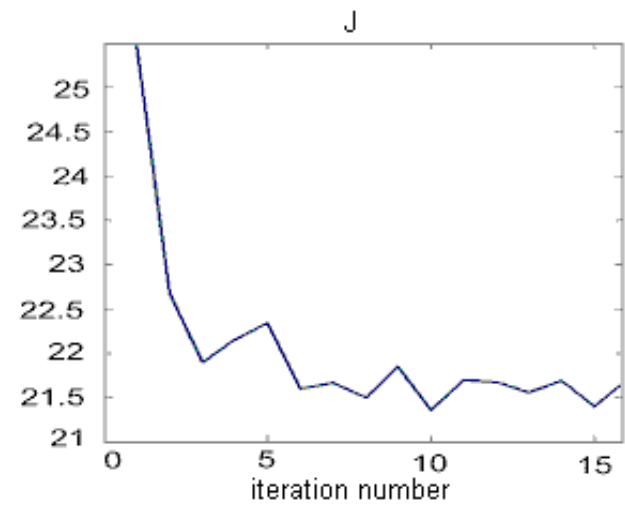

Fig. 7. Objective function versus iteration number

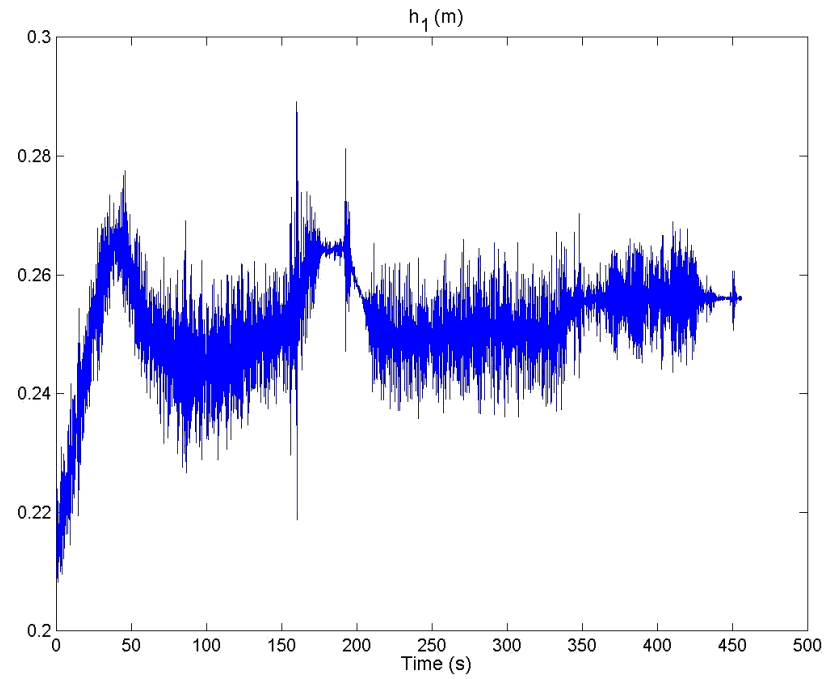

Fig. 8. Real-time experimental results: $h_{1}$ versus time after 12 iterations of the IFT algorithm

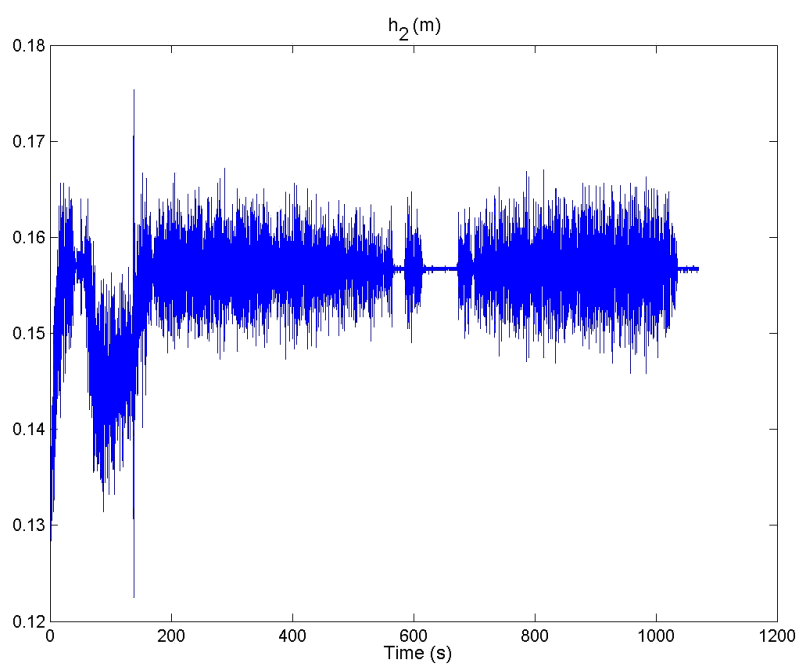

Fig. 9. Real-time experimental results: $h_{2}$ versus time after 12 iterations of the IFT algorithm

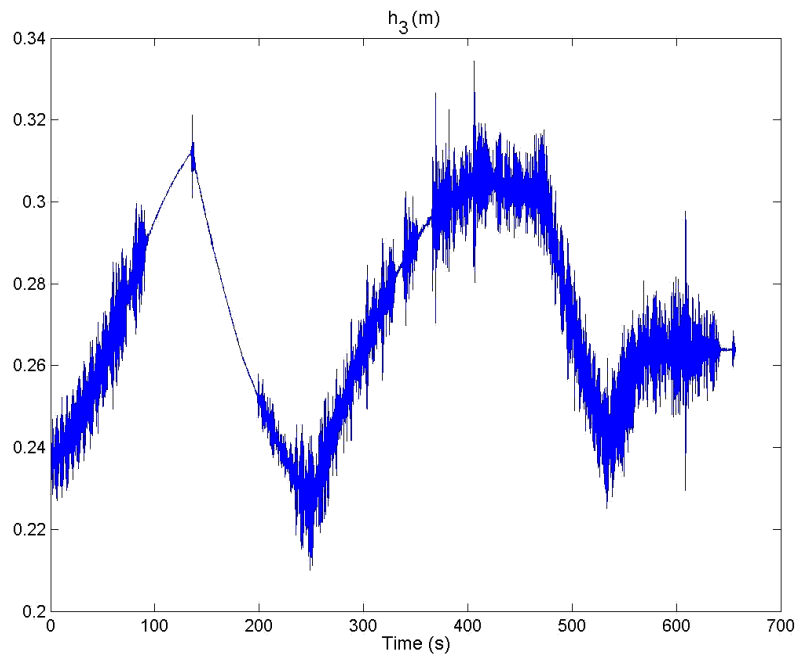

Fig. 10. Real-time experimental results: $h_{3}$ versus time after 12 iterations of the IFT algorithm 


\section{CONCLUSION}

The paper has proposed a new low-cost control solution based on the IFT applied to the level control of the three-tank system laboratory equipment. The digital simulation and realtime experimental results show the CS performance improvement.

Future research will be focused on the convergence analysis of IFT algorithms. All theoretical results will be tested in the control of complex processes that involve also fuzzy controllers [27]-[34].

\section{ACKNOWLEDGMENT}

The paper was supported by the CNMP and CNCSIS of Romania, the "Politehnica" University of Timisoara, the Óbuda University, Budapest, Hungary, and the University of Ljubljana, Slovenia, from the Hungarian-Romanian and Slovenian-Romanian Intergovernmental $S$ \& $T$ Cooperation Programs. This work was partially supported by the strategic grant POSDRU 6/1.5/S/13 (2008) of the Ministry of Labor, Family and Social Protection, Romania, co-financed by the European Social Fund - Investing in People.

\section{REFERENCES}

[1] Z. Petres, P. Baranyi, P. Korondi, and H. Hashimoto, "Trajectory tracking by TP model transformation: case study of a benchmark problem," IEEE Trans. Ind. Electron., vol. 54, pp. 1654-1663, June 2007.

[2] O. Gauci, C. J. Debono, and P. Micallef, "An enhanced centered binary tree of SVMs algorithm for phoneme recognition," in Proc. EUROCON, Warsaw, Poland, 2007, pp. 209-213.

[3] B. Dumnic, Z. Ivanovic, V. Katic, V. Vasic, and M. Delimar, "Sensorless vector control and effects of machine parameters mismatch in variable speed wind turbines," in Proc. EUROCON, Warsaw, Poland, 2007, pp. 1654-1659.

[4] J. D. Hewlett, B. M. Wilamowski, and G. Dundar, "Optimization using a modified second-order approach with evolutionary enhancement," IEEE Trans. Ind. Electron., vol. 55, pp. 3374-3380, Sept. 2008.

[5] P. Cortes, M. P. Kazmierkowski, R. M. Kennel, D. E. Quevedo, and J. Rodriguez, "Predictive control in power electronics and drives," IEEE Trans. Ind. Electron., vol. 55, pp. 4312-4324, Dec. 2008.

[6] D. Bauso, L. Giarre, amd R. Pesenti, "Robust control of uncertain multi-inventory systems via linear matrix inequality," in Proc. 2008 American Control Conference, Seattle, WA, 2008, pp. 4081-4086.

[7] S. H. Kia, H. Henao, and G.-A. Capolino, "Diagnosis of broken-bar fault in induction machines using discrete wavelet transform without slip estimation," IEEE Trans. Ind. Appl., vol. 45, pp. 1395-1404, Aug. 2009.

[8] Y. Xinghuo and O. Kaynak, "Sliding-mode control with soft computing: a survey," IEEE Trans. Ind. Electron., vol. 56, pp. 32753285, Sept. 2009.

[9] H. Hjalmarsson, S. Gunnarsson, and M. Gevers, "A convergent iterative restricted complexity control design scheme," in Proc. $33^{\text {rd }}$ IEEE Conference on Decision and Control (CDC), Lake Buena Vista, FL, 1994, pp. 1735-1740.

[10] H. Hjalmarsson, "Iterative feedback tuning - an overview," Int. J. Adapt. Control Signal Process., vol. 16, pp. 373-395, May 2002.

[11] J. K. Huusom, N. K. Poulsen, and S. B. Jørgensen, "Improving convergence of iterative feedback tuning," J. Process Control, vol. 19, pp. 570-578, Apr. 2009.

[12] H. Hjalmarsson and T. Birkeland, "Iterative feedback tuning of linear time-invariant MIMO systems," in Proc. $37^{\text {th }}$ IEEE Conference on Decision and Control (CDC), Tampa, FL, 1998, pp. 3893-3898.

[13] H. Hjalmarsson, "Efficient tuning of linear multivariable controllers using iterative feedback tuning," Int. J. Adapt. Control Signal Process., vol. 13, pp. 553-572, Nov. 1999.
[14] H. Jansson and H. Hjalmarsson, "Gradient approximations in iterative feedback tuning for multivariable processes," Int. J. Adapt. Control Signal Process., vol. 18, pp. 665-681, Oct. 2004.

[15] R. -E. Precup, S. Preitl, I. J. Rudas, M. L. Tomescu, and J. K. Tar, "Design and experiments for a class of fuzzy controlled servo systems," IEEE/ASME Trans. Mechatronics, vol. 13, pp. 22-35, Feb. 2008.

[16] S. Kissling, P. Blanc, P. Myszkorowski, and I. Vaclavik, “Application of iterative feedback tuning (IFT) to speed and position control of a servo drive," Control Eng. Pract., vol. 17, pp. 834-840, Jul. 2009.

[17] J. K. Huusom, N. K. Poulsen, and S. B. Jørgensen, "Data driven tuning of state space control loops with unknown state information and model uncertainty," Comput. Aided Chem. Eng., vol. 26, pp. 441-446, Jan. 2009.

[18] R. Dormido, H. Vargas, N. Duro, J. Sanchez, S. Dormido-Canto, G. Farias, F. Esquembre, and S. Dormido, "Development of a web-based control laboratory for automation technicians: the three-tank system," IEEE Trans. Educ., vol. 51, pp. 35-44, Feb. 2008.

[19] B. Bouzouita, F. Bouani, W. Wertz, and M. Ksouri, "Implementation of SISO robust predictive control to a three tanks system," in Proc. IEEE International Conference on Control Applications (CCA 2008), San Antonio, TX, 2008, pp. 323-328.

[20] L. F. Mendonca, J. Sousa, and J. M. G. Sa da Costa, "Fault accommodation of an experimental three tank system using fuzzy predictive control," in Proc. IEEE International Conference on Fuzzy Systems (FUZZ-IEEE 2008), Hong Kong, 2008, pp. 1619-1625.

[21] R. Benayache, L. Chrifi-Alaoui, P. Bussy, and J. M. Castelain, "Nonlinear sliding mode control with backstepping approach for a nonlinear three tank system," in Proc. $16^{\text {th }}$ Mediterranean Conference on Control and Automation, Ajaccio, France, 2008, pp. 658-663.

[22] N. Orani, A. Pisano, and E. Usai, "Fault detection and reconstruction for a three-tank system via high-order sliding-mode observer," in Proc. IEEE International Conference on Control Applications (CCA '09), Saint Petersburg, Russia, 2009, pp. 1714-1719.

[23] Amira, "DTS200 - laboratory setup three tank system," Amira GmbH, Duisburg, Germany, 2000.

[24] I. Mosincat, "Iterative feedback tuning techniques: applications to control or three-tank system," B.Sc. thesis, Dept. Autom. Appl. Inform., "Politehnica" Univ. of Timisoara, Timisoara, Romania, 2009.

[25] R.-E. Precup and S. Preitl, "Development method for low cost fuzzy controlled servosystems," in Proc. 2006 IEEE International Symposium on Intelligent Control (ISIC), München, Germany, 2006, pp. 2707-2712.

[26] M.-B. Rădac, R.-E. Precup, S. Preitl, J. K. Tar, and K. Burnham, “Tire slip fuzzy control of a laboratory anti-lock braking system," in Proc. European Control Conference 2009 (ECC '09), Budapest, Hungary, 2009, pp. 940-945.

[27] L. Horváth and I. J. Rudas, Modelling and Solving Methods for Engineers. Burlington, MA: Elsevier, Academic Press, 2004.

[28] I. Škrjanc, S. Blažič, S. Oblak, and J. Richalet, "An approach to predictive control of multivariable time-delayed plant: Stability and design issues," ISA Transactions, vol. 43, pp. 585-595, Oct. 2004.

[29] Z. C. Johanyák and S. Kovács, "Fuzzy rule interpolation based on polar cuts," in Computational Intelligence, Theory and Applications, B. Reusch, Ed. Berlin, Heidelberg, New York: Springer Verlag, 2006, pp. 499-511.

[30] D. J. N. Limebeer and R. S. Sharp, "Bicycles, motorcycles, and models,” IEEE Control Syst. Mag., vol. 26, pp. 34-61, Oct. 2006.

[31] D. Barrettino, P. Malcovati, M. Graf, S. Hafizovic, and A. Hierlemann, "CMOS-based monolithic controllers for smart sensors comprising micromembranes and microcantilevers," IEEE Trans. Circuits Syst. I, Reg. Papers, vol. 54, pp. 141-152, Jan. 2007.

[32] J. Vašč́k, "Fuzzy cognitive maps in path planning," Acta Technica Jaurinensis, Series Intelligentia Computatorica, vol. 1, pp. 467-479, Dec. 2008.

[33] K. Jezernik, "High precision VSS motion control of servo drives," in Proc. $10^{\text {th }}$ IEEE International Workshop on Advanced Motion Control (AMC '08), Trento, Italy, 2008, pp. 710-715.

[34] M. Jones, S. N. Vukosavic, and E. Levi, "Parallel-connected multiphase multidrive systems with single inverter supply," IEEE Trans. Ind. Electron., vol. 56, pp. 2047-2057, June 2009. 